\title{
Alain Pagès, Zola et le groupe de Médan. Histoire d'un cercle littéraire, Paris, Perrin, 2014, 480 p.
}

Jean-Marc Hovasse

\section{(2) OpenEdition}

1 Journals

\section{Édition électronique}

URL : http://journals.openedition.org/genesis/1557

DOI : 10.4000/genesis. 1557

ISSN : 2268-1590

Éditeur :

Presses universitaires de Paris Sorbonne (PUPS), Société internationale de génétique artistique littéraire et scientifique (SIGALES)

Édition imprimée

Date de publication : 27 novembre 2015

Pagination : 155-159

ISBN : 9791023105049

ISSN : 1167-5101

\section{Référence électronique}

Jean-Marc Hovasse, «Alain Pagès, Zola et le groupe de Médan. Histoire d'un cercle littéraire, Paris, Perrin, 2014, 480 p. », Genesis [En ligne], 41 | 2015, mis en ligne le 03 mai 2017, consulté le 22 septembre 2020. URL : http://journals.openedition.org/genesis/1557 ; DOI : https://doi.org/10.4000/genesis. 1557 


\section{Comptes rendus d'ouvrages sur la création collaborative}

\begin{abstract}
Alain Pagès, Zola et le groupe de Médan. Histoire d'un cercle littéraire, Paris, Perrin, 2014, 480 p.
\end{abstract}

\section{Compte rendu par Jean-Marc Hovasse}

Le récit «met en scène une aventure collective », annonce la quatrième de couverture de ce beau livre soustitré Histoire d'un cercle littéraire - « essai de biographie collective » ajoute Alain Pagès dans l'envoi réservé à l'auteur de ces lignes, qui n'en ferait pas état si tout cela ne convergeait pour désigner l'angle d'étude de ce compte rendu, en harmonie avec le thème du numéro de Genesis sur la création collaborative.

Fils unique, Zola ne semblait pas a priori désigné pour devenir le centre d'une aventure créatrice collective. D'où l'importance de remonter à sa jeunesse, et à l'exceptionnelle amitié fraternelle qui le réunit dès ses premières années de collège à Aix-en-Provence à Paul Cézanne et à Jean-Baptistin Baille. Trio d'inséparables marqué par des baignades en plein soleil, dans une nature généreuse, où éclosent ensemble deux artistes de tout premier plan, et un polytechnicien. Le parallèle s'impose dès lors entre les souvenirs de Zola, exploités entre autres dans La Confession de Claude, son premier roman publié l'année de ses 25 ans (1865) puis dans L'Euvre (1886), et ceux de Cézanne à l'origine de la série des Baigneurs puis des Baigneuses : "Le décor est identique. Une même vision se retrouve, et surtout une même volonté de reproduire la matière du souvenir pour la retravailler sans cesse. » Il ne s'agit certes pas là de création collective, mais d'œuvres individuelles à partir d'une expérience et de souvenirs communs. C'est du reste bien ainsi que Zola semble l'entendre : s'il a la solitude en horreur (Alain Pagès insiste justement sur les confidences envoyées à Baille dans la lettre du 14 mai 1860), il n'a jamais imaginé au départ de collaboration autre que complémentaire, c'est-à-dire entre représentants d'arts différents. Le 25 mars 1860, il avait écrit à Cézanne : «J'ai fait un rêve, l'autre jour. J'avais écrit un beau livre, un livre sublime que tu avais illustré de belles, de sublimes gravures. Nos deux noms en lettres d'or brillaient, unis sur le premier feuillet, et, dans cette fraternité du génie, passaient inséparables à la postérité. Ce n'est encore qu'un rêve malheureusement. » Cette œuvre de rêve pour laquelle les bibliophiles du futur se seraient damnés, l'auteur du Rêve et de L'Euvre ne la réalisera jamais. Reste cette association initiale de la littérature et de la peinture, qui se retrouve aussi, à une échelle peut-être moindre, au début de la carrière de Victor Hugo, avec l'ami Louis Boulanger, mais aussi Célestin Nanteuil et les frères Johannot. L'atelier semble être l'antichambre chronologique, et même logique, du cénacle.

C'est toujours par l'amitié de Cézanne et de Zola qu'Alain Pagès, très sensible dans ce volume à la question des arts, illustre les premiers cénacles naturalistes. Il le fait plus précisément à partir de deux tableaux qui mettent en scène un ami aixois du peintre, jeune auteur monté à Paris rencontrer le romancier qui a sept ans de plus que lui et qu'il a pour modèle dans la vie : Paul Alexis lisant à Émile Zola (Musée des beaux-arts de São Paulo, fig. 1) et Une lecture de Paul Alexis chez Zola (collection particulière, ancienne collection Zola, fig. 2). Lectures indissociables de l'écriture : ce ne sont pas des livres qui sont lus, mais des manuscrits, et les deux tableaux montrent à côté des auteurs presque siamois à l'œuvre leurs outils de travail, la plume et l'encrier. Ce sont en quelque sorte des représentations, sinon des allégories, de la création collaborative : 


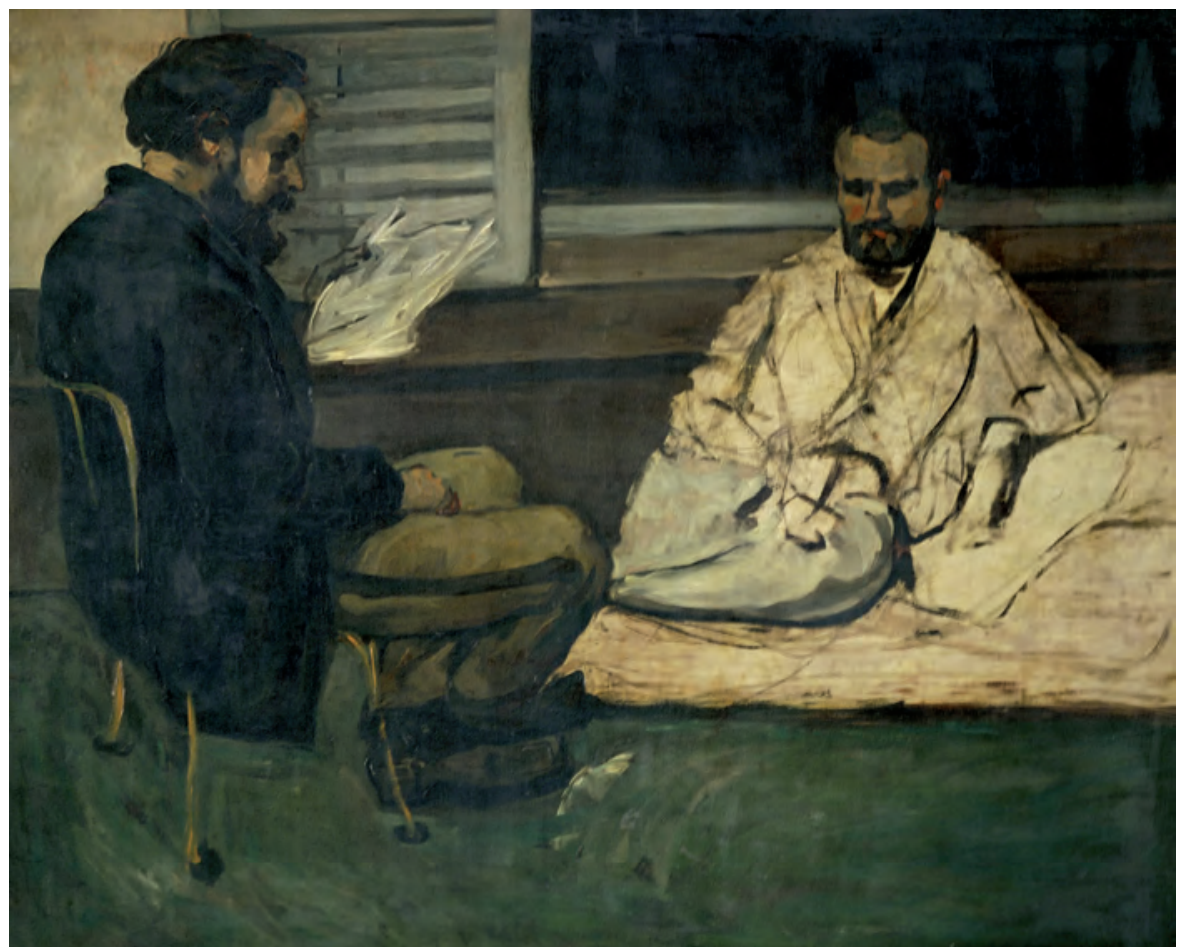

Fig. 1 : Paul Cézanne, Paul Alexis lisant à Émile Zola, 1869-1870

Huile sur toile, $130 \times 160 \mathrm{~cm}$ (Musée d'art de São Paulo, Brésil) akg-images/Erich Lessing

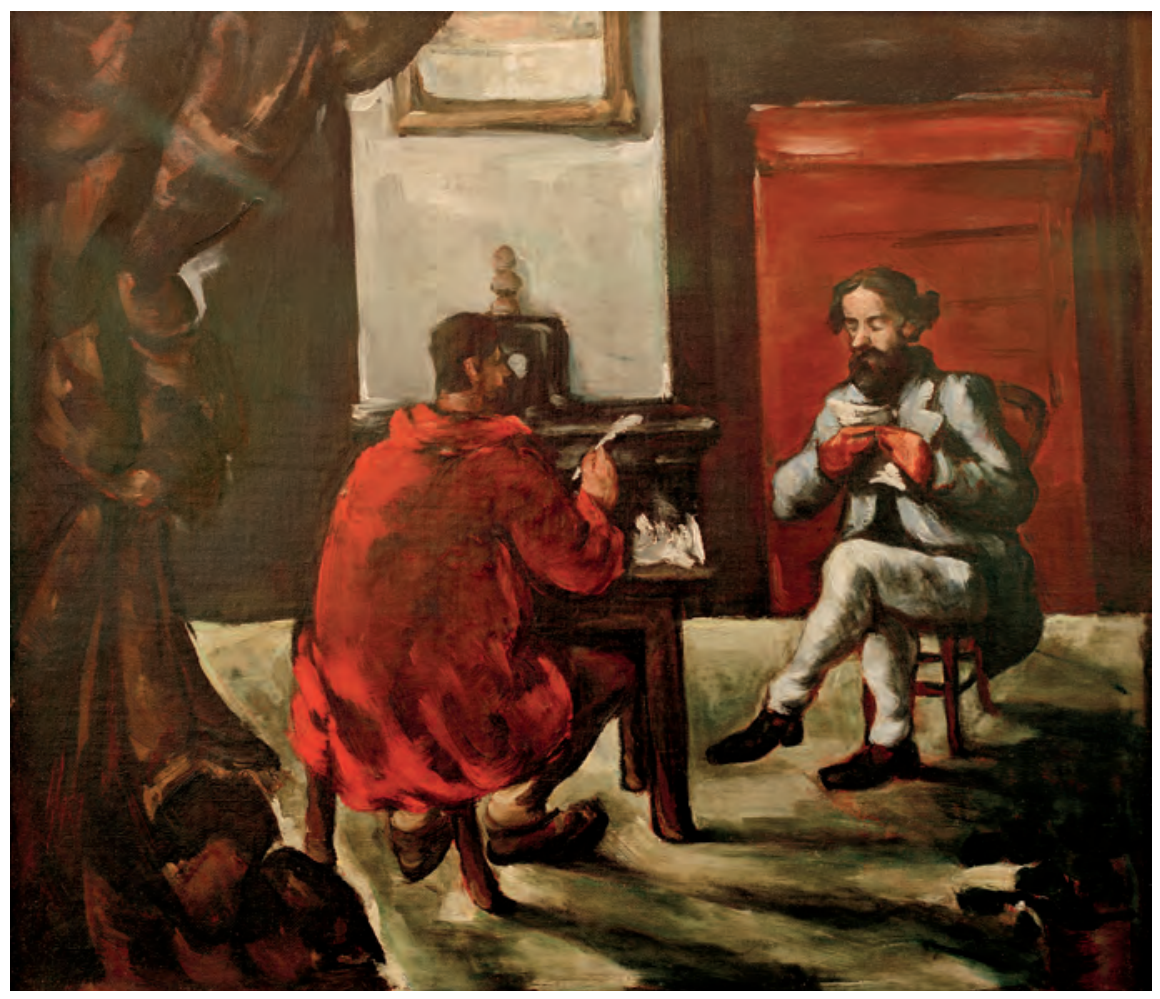

Fig. 2 : Paul Cézanne, Une lecture de Paul Alexis chez Zola, 1869 Huile sur toile, $52 \times 56 \mathrm{~cm}$ (coll. particulière) akg-images 
Le manuscrit que les deux écrivains déchiffrent constitue une première esquisse d'un texte qui sera repris et transformé, après avoir été discuté. La scène se situe aux antipodes d'une peinture romantique qui célébrerait le caractère divin de l'inspiration poétique. À l'image traditionnelle du génie solitaire s'oppose la vision moderne du travail de l'écriture, d'une réflexion conduite en commun, où chacun s'efforce d'aider l'autre. Au fond, il importe peu de savoir qui lit et qui écoute. Les rôles sont interchangeables. [...] Ce que Cézanne a voulu illustrer - dans une recherche picturale qui va bien au-delà de ce que représentent Manet et Fantin-Latour à la même époque -, c'est une certaine idée de la collaboration intellectuelle, telle qu'elle est vécue au sein du cénacle des Batignolles. Ni maîtres, ni disciples, mais des amis qui s'épaulent mutuellement avec l'espoir d'imposer collectivement leur présence sur la scène littéraire.

On songe en lisant cette description aux frères Goncourt, lesquels paieront du reste assez mal l'admiration initiale que leur avait vouée le jeune Émile Zola. Que ce soit pour le journal (l'aventure de l'éphémère Marseillaise au début de l'année terrible), au théâtre ou chez lui, ce dernier ne met jamais en doute la devise de la Belgique selon laquelle c'est l'union qui fait la force. Confrères d'Aix-en-Provence montés à Paris, jeunes admirateurs de son œuvre, il ne néglige rien ni personne dans son ascension progressive, que le scandale de L'Assommoir accélère brutalement. D'où l'importance de fédérer ceux qui sont prêts à se battre pour lui, un peu à l'image des aficionados d'Hernani en 1830. La lutte, dépourvue d'arène théâtrale jusqu'à l'adaptation du roman à l'Ambigu en janvier 1879, est moins spectaculaire, mais à peine moins violente. Les combattants principaux, présentés un par un par Alain Pagès, qui dans sa veine picturale soigne naturellement sa galerie de portraits, finissent par se regrouper dans un club des cinq : Paul Alexis, Maupassant, Huysmans, Henry Céard et Léon Hennique. « À cinq on peut bien des choses, et peut-être y a-t-il des trucs inusités jusqu'ici », avait écrit Maupassant à Paul Alexis le 17 janvier 1877 ; métaphore canaille préparée par une incise de la même lettre rétablie en 2012 seulement par Marlo Johnston dans sa biographie de Maupassant, reprise par Alain Pagès : « Pourquoi s'interdire de baiser autrement qu'à l'épicière ou qu'à la gamin, quand il existe tant de positions et quand nous avons le devoir d'en découvrir de nouvelles ? » Zola qui vient d'acheter sa mai- son de Médan grâce aux droits de L'Assommoir fédère le mouvement avec application. Envoyant des remontrances à tel ou tel de ses disciples qui semble ne pas travailler assez, il lui rappelle : « Nous devons d'ici à quelques années écraser le public sous notre fécondité » (16 juillet 1877). Les cinq disciples répondent à une invitation à Médan, vers la fin de l'année 1878, par une lettre commune dont l'entête est « Notre cher ami » : telle qu'elle apparaît du moins à travers l'usage d'adjectifs possessifs inusités, la communauté semble étroite. De Zola à la veille du millésime 1880 on pourrait écrire comme de Brahma : «Il ne dit plus : Je suis ! mais il pensa : Nous sommes ${ }^{1}$ ! »

Autour de Zola, rapporte Alain Pagès, existe bien « une sorte d'atelier d'écriture », dont l'hospitalité de Médan forme la pierre angulaire. L'auteur de Nana (1880) met par exemple à contribution Henry Céard, ancien carabin, pour décrire le visage de son héroïne morte de la variole. Mais cette collaboration, assez similaire à celle de Flaubert et de Maupassant pour Bouvard et Pécuchet, va toujours dans le même sens. D'où l'intérêt et l'originalité du projet des Soirées de Médan, par lequel ses disciples proposent à Zola, à la fin de l'année 1879, « une autre forme de collaboration, plus égalitaire, fondée non sur la fusion des écritures, mais sur une juxtaposition de textes autonomes ». Nouvelles complémentaires unifiées par leur sujet, la guerre de 1870 , et par leur traitement naturaliste qui refuse le patriotisme cocardier qui avait été de règle jusque-là pour offrir un contrepoids au désastre de Sedan et à ses conséquences. Flaubert, qui meurt l'année de leur publication, avait eu le temps d'en suivre la genèse, et de juger « stupide » leur titre collectif - cet hommage à Zola et à son hospitalité ayant été finalement préféré à L'Invasion comique. Sans doute n'avait-il pas entendu, derrière Les Soirées de Médan, une contrepèterie presque parfaite, aux accents près, en tout cas hautement significative (Les Moires de Sedan). Quoi qu'il en soit, trois nouvelles ayant été déjà publiées dans diverses revues, il s'agissait, contrairement à la légende créée par les auteurs eux-mêmes, moins d'une véritable création collaborative que d'une manifestation de force, le groupe s'étant surtout réuni pour décider du titre, de l'ordre des nouvelles (Zola et son Attaque du moulin

1. Leconte de Lisle, «La Vision de Brahma », Revue contemporaine, 15 avril 1857, repris dans les Poèmes antiques de 1874. 
en premier, les autres tirés au sort), et de la brève préface datée du 1er mars 1880 qui se termine par la phrase suivante : « Notre seul souci a été d'affirmer publiquement nos véritables amitiés et, en même temps, nos tendances littéraires. »

Ironie du sort, cette œuvre collective à la gloire de Zola est finalement restée dans l'histoire littéraire pour avoir lancé Maupassant, dont la nouvelle Boule de suif écrase les cinq autres - sans que Zola n'ait jamais paru s'en formaliser. Sa place lui suffisait, qui rendait jaloux Edmond de Goncourt : «Zola a une gens de jeunes fidèles, dont le malin écrivain entretient et nourrit du reste l'admiration, l'enthousiasme, la flamme par l'octroi de correspondances à l'étranger, le faufilement bien payé dans les journaux où il règne en maître, enfin par des services tout matériels. » Les faits semblent démentir cette vision des choses : Zola ne parvient pas l'année même des Soirées de Médan à soutenir suffisamment la création d'une revue naturaliste, La Comédie humaine, dont Huysmans aurait été le rédacteur en chef. Les premières dissensions apparaissent dans le groupe des cinq dès cette date-là. Le projet avorté d'un Théâtre de Médan (sans contrepèterie dans le titre) ne les réunira pas davantage, même si Huysmans et Hennique iront le plus loin dans la voie déjà bien balisée de la collaboration dramatique en écrivant à deux mains, au début de l'année 1881, la pantomime naturaliste Pierrot sceptique.

La « crise des disciples » va suivre leur ultime apogée, telle qu'elle apparaît dans le dossier de Pot-Bouille (1882), dont une section est explicitement intitulée « Notes de Céard et d'Huysmans ». Le roman naît en effet des conversations entre Zola et ses amis ; les notes sont écrites par Zola lui-même, par Céard, et plusieurs lettres de Huysmans et de Céard complètent le dossier. Alain Pagès lui réserve une page éclairante : «Les écritures manuscrites se succèdent et s'enchevêtrent, écho des idées qui se sont croisées et des propos qui ont été échangés. Ces strates souterraines, enfouies dans le dossier, ont permis le surgissement de la fiction. [...] Un essai d'écriture collaborative qui anticipe sur ce que connaîtra le mouvement surréaliste, bien des années plus tard. »Cas d'école, donc, et non règle de conduite, même si l'on peut noter que ce roman se trouve à peu près au centre géométrique de l'édifice des Rougon-Macquart. À partir du milieu des années 1880 cependant, marquées aussi par la mort de Victor Hugo, Émile Zola paraît sou- dain plus solitaire. Edmond de Goncourt semble avoir repris l'initiative en réunissant dans son Grenier d'Auteuil, avec le concours actif d'Alphonse Daudet, la nouvelle génération montante. Léon Hennique, futur légataire universel de Goncourt avec l'auteur du Petit Chose, sera le transfuge le plus emblématique de Médan à Auteuil. Il faut voir dans L'CEuvre, suggère Alain Pagès, la traduction du désarroi de son auteur, et peut-être moins la simple histoire de ses relations avec Cézanne que « la fin d'une espérance commune qu'incarnait le mythe des Soirées de Médan »: "Chacun des membres de la "bande" s'efforce désormais de se frayer un chemin dans l'existence, en ignorant les solidarités anciennes : et le maître qui présidait autrefois au banquet des lettres assiste, impuissant, à la disparition d'un rêve collectif. » Le coup de grâce arrive l'année suivante, après la publication de La Terre, sous la forme d'un nouveau «Manifeste des Cinq » publié en première page du Figaro le 18 août 1887 : règlement de compte contre « cette imposture de la littérature véridique, cet effort vers la gauloiserie mixte d'un cerveau en mal de succès », signé de cinq noms représentatifs de la nouvelle génération (Paul Bonnetain, J.-H. Rosny, Lucien Descaves, Paul Margueritte et Gustave Guiches). Aucune intersection officielle, heureusement, entre les auteurs des Soirées de Médan et les signataires de cet article « imbécile et ignoble » auquel Zola refuse de répondre directement, mais le mal est fait. Ses conséquences se feront sentir bien au-delà du nouveau siècle qui s'approche.

Les projets littéraires collectifs de Zola sont décidément loin derrière lui, et il ne fait plus rien, ou pas grandchose, pour les relancer. Il préfère pour ses adaptations du Rêve (1891) et surtout de L'Attaque du moulin (1893) traiter directement avec le compositeur Alfred Bruneau (sur cette collaboration réussie, mais de nouveau entre représentants d'arts différents, lire les travaux de JeanSébastien $\mathrm{Macke}^{2}$ ). Il écoute certes les conseils éclairés d'Henry Céard, mais refuse de l'associer plus avant dans le processus créatif, accélérant ainsi le détachement puis la rancune de cet ancien disciple féru de musique. Et puis

2. Pour un théâtre lyrique naturaliste, thèse de doctorat, Université de Reims, 2003 ; «L'Attaque du moulin : genèse d'un opéra d'Alfred Bruneau d'après une nouvelle d'Émile Zola », Genesis, n 40, 2015, p. $155-170$. 
sa vie sentimentale contribue à le recentrer sur lui-même : sa liaison avec Jeanne Rozerot, qui lui donne deux enfants avant que son épouse Alexandrine ne l'apprenne, déclenchant une grave crise conjugale dont on n'a pu prendre que tout récemment la mesure ${ }^{3}$, renvoie dans un passé très lointain les années idéales de Médan, où Alexandrine recevait les jeunes amis de son mari comme les enfants qu'ils n'avaient pas eus. Il faudra attendre l'affaire Dreyfus, nouvelle et ultime lutte collective abordée par Zola en solitaire, pour permettre à l'auteur de L'Assommoir de retrouver en quelque sorte sa place dans le paysage littéraire contemporain. Mais il paiera cette reconquête au prix fort : l'exil, et peut-être la mort.

Avec Zola et le groupe de Médan, Alain Pagès a réussi son pari d'une biographie collective, élégamment composée et servie par une écriture claire. Même si l'on ne voit que Zola (à sa table de travail, par Nadar) sur sa magnifique couverture qui compense par ailleurs l'absence regrettable d'autres illustrations, son livre muni de toutes les références nécessaires, d'une bibliographie complète et d'un index précieux, traverse trois quarts de siècle avec la majesté tranquille de la Seine à Médan. Très en amont des méandres yvelinois, il remonte à sa source première, les années 1850 en Provence, et descend jusqu'à son embouchure et même au-delà : l'épilogue raconte le cinquantième anniversaire de la publication des Soirées de Médan, célébré le 3 mai 1930 par un banquet sous la présidence de Léon Hennique, le dernier survivant des six, au restaurant Édon (renommé deux ans plus tard « Au bœuf couronné », toujours ouvert au 188, avenue Jean-Jaurès à Paris). « Le romancier vit la création littéraire comme une expérience de groupe, en faisant de l'amitié un moteur de l'écriture », annonçait la quatrième de couverture. Il y a loin toutefois de cet idéal, conçu dans l'exaltation de la jeunesse puis approché au plus près selon deux modalités différentes au début des années 1880 dans Les Soirées de Médan et dans Pot-Bouille, à la réalité d'une certaine solitude créatrice éprouvée assez vite et surtout dans l'âge mûr. C'est un peu ce parcours du combattant que permet de suivre le récit où se dessine à petites touches, au milieu de ses amis, un portrait de Zola qui finalement le grandit : énergique et généreux, ouvert et concentré, naïf et sensible, peu rancunier, toujours soucieux d'apaisement, il n'est pas vraiment responsable de l'échec de son idéal. La faute, si faute il y a, doit être recherchée dans son entourage - le genus irritabile vatum se prête assez mal au travail d'atelier plus naturel chez les peintres -, ou dans la vie elle-même. Comme le notaient déjà Anthony Glinoer et Vincent Laisney à la fin de leur ouvrage sur les Confraternités littéraires et artistiques au XIXe siècle, "le combat est collectif, mais la gloire est individuelle ${ }^{»}$ ». C'est la raison pour laquelle les anciens combattants ont de meilleurs souvenirs des mêlées que des victoires remportées par l'un d'eux. En évoquant dans Le Journal du 31 janvier 1893 le troquet de la mère Machini, dit «L'Assommoir », à l'angle de la rue Coustou et de la rue Puget, disparu celui-là mais où se trouvait encore il y a peu une boîte de strip-tease assez louche, Paul Alexis n'écrivait pas autre chose. Comme il songeait alors sans doute aussi aux soirées de Médan, et peut-être plus largement encore à toutes les aventures littéraires de création collaborative, ce disciple, qui avait « deux prénoms et pas de nom » (Félicien Champsaur) et que son confrère Henry Céard avait conséquemment rebaptisé l' « ombre de Zola »-destin un peu tragique pour un ancien coauteur, à peine compensé par l'immortalité que Cézanne lui avait conférée à ses côtés - aura pour une fois le dernier mot :

Quelle époque heureuse, lorsqu'on la revoit en pensée, que cette heure des débuts ! Plus tard, une fois dans la bataille, chacun va de son côté, songe à sa peau, et livre son combat personnel. On ne se rencontre plus que de loin en loin [...] ; puis on repart, chacun dans sa direction - peut-être pour ne jamais se revoir. - Aussi le meilleur temps est-il celui des débuts, où l'on ne se perd pas de vue, parce que le rêve est plus pur, plus complet que la réalité, que l'action, et qu'on se contente encore de caresser en commun un beau rêve.

3.Lettres à Alexandrine, éd. Brigitte Émile-Zola et Alain Pagès, Paris, Gallimard, 2014.

4. L'Âge des cénacles. Confraternités littéraires et artistiques au XIXe siècle, Paris, Fayard, 2013, p. 555. 